\title{
TEMPO LIVRE E USO DE ÁLCOOL E OUTRAS DROGAS: ESTUDO COMPARATIVO ENTRE ESTUDANTES UNIVERSITÁRIOS DO BRASIL E PORTUGAL
}

\author{
FREE TIME AND USE OF ALCOHOL AND OTHER DRUGS: A STUDY BETWEEN \\ BRAZILIAN AND PORTUGUESE UNDERGRADUATE STUDENTS
}

\author{
TIEMPO LIBRE Y CONSUMO DE ALCOHOL Y OTRAS DROGAS: UN ESTUDIO \\ COMPARATIVO ENTRE ESTUDIANTES UNIVERSITARIOS DE BRASIL Y \\ PORTUGAL
}

\section{Liana Abrão Romera*, Raul Aragão Martins *, Heloisa Heringer Freitas*, Derick dos Santos Tinoco*, Regina Cassia Rondina**}

Palavras chave:

Consumo de álcool na faculdade. Uso de drogas. Atividades de lazer. Esportes.
Resumo: O tempo livre está presente em todas as fases da vida e geralmente é associado ao lazer, que entre os jovens muitas vezes é acompanhado do uso de álcool e outras drogas. Investigar a forma como estudantes universitários utilizam este tempo e qual o padrão de consumo de drogas legais e ilegais é o objetivo deste trabalho, que analisa universitários brasileiros e portugueses. Participaram 238 jovens, sendo 60,5\% deles portugueses, que apresentaram a média de idade de 19,9 anos. Resultados mostram que praticar esportes $(31,0 \%)$, sair com amigos $(18,8 \%)$ e namorar $(13,4 \%)$ são as três atividades mais citadas. Bebidas alcoólicas e 0 tabaco são as drogas mais utilizadas, citadas por mais de $50 \%$ dos estudantes, seguidas de maconha, com $15,9 \%$ de usuários. Estudantes brasileiros apresentam padrão de beber excessivo maior do que os portugueses. Concluímos que estes estudantes praticam esportes, fato que não impede o uso de álcool.

Keywords: Alcohol drinking in college.

Drug use.

Leisure activities. Sports.

Palabras clave: Consumo de alcohol en la universidad. Uso de drogas. Actividades recreativas. Deportes.

\begin{abstract}
Free time is an integral part of all life stages and is generally associated with leisure, which among youth often comes with the use of alcohol and drugs. The way undergraduate students from Portugal and Brazil use their free time and their patterns of legal and illegal drug consumption are the objectives of this work. The sample included 238 undergraduate students $-60.5 \%$ of whom were Portuguese, with an average age of 19.9. Results show that sports practice (31.0\%); going out with friends (18.8\%); and dating $(13.4 \%)$ were the three most common activities. Alcoholic beverages and tobacco are the drugs most often mentioned by $50 \%$ of the sample, and marijuana use was reported by $15.9 \%$. Heavy alcohol drinking patterns are more common among Brazilian undergraduate students than among Portuguese ones. In conclusion, we stress that these students practice sports but that it do not inhibit alcohol consumption.
\end{abstract}

Resumen: El tiempo libre está en todas fases de la vida, generalmente se asocia al ocio, que entre los jóvenes muchas veces acompaña el uso de alcohol y drogas. La forma en que los estudiantes universitarios utilizan ese tiempo y cuál es el patrón de consumo de drogas legales e ilegales es el objetivo de este trabajo, que investiga universitarios brasileños y portugueses. Participaron 238 jóvenes, $60,5 \%$ de ellos portugueses, con edad media de 19,9 años. Los resultados muestran que practicar deportes (31,0\%), salir con amigos (18,8\%) y salir con una pareja $(13,4 \%)$, son las tres actividades más citadas. Las bebidas alcohólicas y el tabaco son las drogas más utilizadas, citadas por más del $50 \%$ de los estudiantes; luego viene la marihuana, con un $15,9 \%$ de usuarios. Brasileños tienen un nivel más elevado que los portugueses de consumo excesivo de alcohol. Concluimos que estos estudiantes practican deportes, hecho que no impide el uso de alcohol.
*Universidade Federal do Espírito Santo. Vitória, ES, Brasil. E-mail: liromera@uol.com.br; heloheringer@gmail.com; dericktinoco@hotmail.com

**Universidade Estadual Paulista. São Carlos, SP, Brasil.

E-mail: raul@ibilce.unesp.br; rcassiar@marilia.unesp.br

Recebido em: 12-04-2018 Aprovado em: 06-06-2018

DOI: https://doi.org/10.22456/1982-8918.81951 (c) (1) 으 Licence 


\section{INTRODUÇÃO}

O consumo de drogas é um fenômeno presente em diferentes culturas e sociedades, representa um hábito antigo que se conserva por muitos motivos, tais como medicinais, religiosos, espirituais e recreacionais. Apesar da estreita relação histórica e cultural que a humanidade preserva com o consumo de drogas, os modos emergentes de uso, especialmente aqueles praticados na esfera do lazer, como o uso recreativo, suscitam nas últimas décadas diferentes olhares e entendimentos (CALDWELL; SMITH, 2006; FREIRE, 2013).

Dentre as principais modificações verificadas na sociedade atual, interessa-nos, nesta pesquisa, aquelas promovidas nos modos de ocupação e consumo durante o lazer da parcela populacional jovem brasileira e portuguesa. Investigações de cunho sociológico têm confirmado o aumento do número de usuários de drogas com fins recreativos, a ampliação na diversidade de substâncias oferecidas, a mudança nos contextos de uso e o impacto das propagandas nos modos de consumo juvenis.

Os novos modos de uso de drogas exigem uma compreensão distanciada da visão maniqueísta do usuário ou sua associação à marginalidade, reforçadora de estigmas e preconceitos desnecessários. Além disso, nas últimas décadas, novas formas de consumo surgiram impulsionadas pelas mudanças econômicas, políticas e culturais provocadas pelo capitalismo ALDRIDGE, MEASHAM; WILLIANS, 1998; FAR et al., 2007; ROMERA; MARTINS; REIS, 2017). A mercantilização das necessidades no mundo globalizado e 0 ethos da alegria reforçam o culto às sensações e prazeres imediatos, o que Bauman (2003) chama de sociedade líquida, sem vínculos. 0 jovem é percebido como integrante de um universo que fomenta o consumo em várias facetas e é marcado pela procura constante por sensações e sentidos, materializadas frequentemente nos contextos recreativos, como nas vivências de tempo livre. Passamos a dispor de mais tempo livre, expandiram-se as necessidades, modificaram-se os valores e as atitudes, a qualidade de vida e a expressão de si se tornaram essenciais. Lipovetsky argumenta que nos encontramos hoje "em uma dimensão hedonista do consumo" (2007, p. 61), na qual o lazer aparece como uma marca fundamental da sociedade.

Para melhor compreender essas formas de diversão, imprime-se a necessidade de pesquisas que considerem o lazer como lócus de investigações para a compreensão dos modos de consumo de substâncias lícitas e ilícitas por parte de uma parcela da população jovem. Nesta perspectiva, este estudo tem o objetivo investigar como estudantes universitários brasileiros e portugueses usam o tempo livre, qual o padrão de consumo de álcool e outras drogas e as relações entre tempo livre e uso de drogas.

\section{MÉTODO}

Estudo quantitativo de caráter comparativo e experimental, com a realização de levantamento das condutas e dos modos de vivência do lazer e uso de drogas em um grupo de universitários brasileiros e portugueses. Ele foi desenvolvido nos cursos de Educação Física das seguintes instituições de ensino superior: no Brasil na Universidade Federal do Espírito Santo na cidade de Vitória, mais especificamente no Centro de Educação Física e 
Desportos; e em Portugal, na Faculdade de Motricidade Humana em Lisboa. A seleção das amostras em ambos os países se deu por acessibilidade e intencionalidade.

Os dados aqui analisados foram coletados com a utilização de um questionário de autopreenchimento e anônimo. 0 anonimato no preenchimento do questionário visava garantir a privacidade dos participantes, dando-Ihes maior liberdade para expressar opinião e modos de vivências e consumos de substâncias psicoativas no lazer. O questionário foi especialmente elaborado para o presente estudo e organizado com questões sobre o perfil sociodemográfico, padrão de exercícios físicos e uso de álcool e outras drogas. O instrumento avalia se os participantes utilizam bebida alcoólica, tipo de bebida, em quais situações, companhias e motivações, situações de iniciação do uso, local de uso, sensação relacionada ao uso e também as dez questões do AUDIT - Teste de Identificação de Desordens Devido ao Uso de Álcool (BABOR et al., 2001), que é um instrumento padronizado para avaliação do uso de álcool desenvolvido pela Organização Mundial de Saúde (OMS) e adaptado para o Brasil por Méndez (1999). O questionário foi aplicado de forma coletiva, durante as aulas dos universitários dos cursos de Educação Física das duas universidades, a saber, no Brasil e em Portugal.

Este estudo utilizou método quantitativo com um grupo composto por 239 estudantes universitários, 145 portugueses $(60,7 \%)$ e 94 brasileiros (39,3\%). Esse grupo ficou constituído por 120 mulheres $(50,2 \%)$ e 118 homens (49,4\%), não havendo diferença significante na distribuição de homens e mulheres entre brasileiros e portugueses. A diferença em relação ao grupo total se deve a um dos participantes não ter assinalado seu sexo no questionário. Os estudantes estavam com idades entre 16 e 46 anos, com 91,2\% deles tendo até 23 anos de idade. A média de idade deles é de 19,92 (DP = 3,71), não tendo diferença significante nas variáveis sexo e nacionalidade.

Foi garantida a livre adesão dos estudantes para a participação neste estudo, bem como 0 anonimato dos participantes no preenchimento do questionário formado por questões abertas e fechadas. Não houve, por parte dos estudantes, qualquer rejeição em participar do estudo em ambos os países. Junto à folha de perguntas foi entregue o Termo de Consentimento Livre e Esclarecido (TCLE) e, ao final do preenchimento do questionário, bem como do TCLE, os participantes depositavam ambas as folhas, questionário e termo, em envelopes correspondentes, garantindo a sua não identificação.

Os resultados das entrevistas foram digitados em um banco de dados especialmente desenvolvido para este estudo e transportados, num segundo momento, para um programa de análises estatísticas (SPSS, 2005), no qual foram computadas as frequências de cada uma das questões e realizadas análises comparativas (PEREIRA, 1999).

\section{RESULTADOS E DISCUSSÃO}

Apresentaremos inicialmente os resultados de como os jovens deste estudo ocupam o seu tempo livre. Como vários deles registraram mais de uma atividade, apresentamos as três atividades mais citadas e, posteriormente, exporemos o uso de álcool e outras drogas e as relações entre estes dois conjuntos de variáveis, drogas e tempo livre. 


\subsection{0 uso do tempo livre}

O grupo como um todo é praticante de esportes, pois $78,7 \%$ responderam positivamente à questão "Você pratica esporte?", tendo sido citados 16 esportes, com o futebol o mais praticado, esta modalidade esportiva foi assinalada por 31,4\% dos participantes. Os demais esportes tiveram no máximo $5,9 \%$ de adesões, como citado para a natação. Mielke et al. (2010), utilizando um instrumento padronizado para avaliar atividade física em alunos do primeiro ano de uma universidade da Região Sul do Brasil, constataram que 57,1\% dos estudantes foram considerados ativos no domínio do lazer, $59,1 \%$ no domínio deslocamentos de um ponto a outro da cidade e $84,4 \%$ no conjunto das duas atividades, mostrando padrão semelhante ao encontrado neste estudo.

Os estudantes relacionaram 22 diferentes atividades, aparecendo praticar esportes $(31,0 \%)$, sair com amigos (18,8\%) e namorar $(13,4 \%)$, como as três atividades mais citadas como primeira opção. As três atividades mais citadas na segunda opção foram nas duas primeiras posições as mesmas da primeira opção, agora com outros porcentuais: praticar esportes $(23,8 \%)$, sair com amigos $(19,2)$ e utilizar a internet $(8,8)$. A atividade mais citada na terceira opção foi sair com amigos (17,6\%), seguida de utilizar internet $(10,5 \%)$ e ler $(9,2 \%)$ (Figura 1).

Figura 1 - Porcentagem das atividades realizadas no tempo livre por ordem de opção

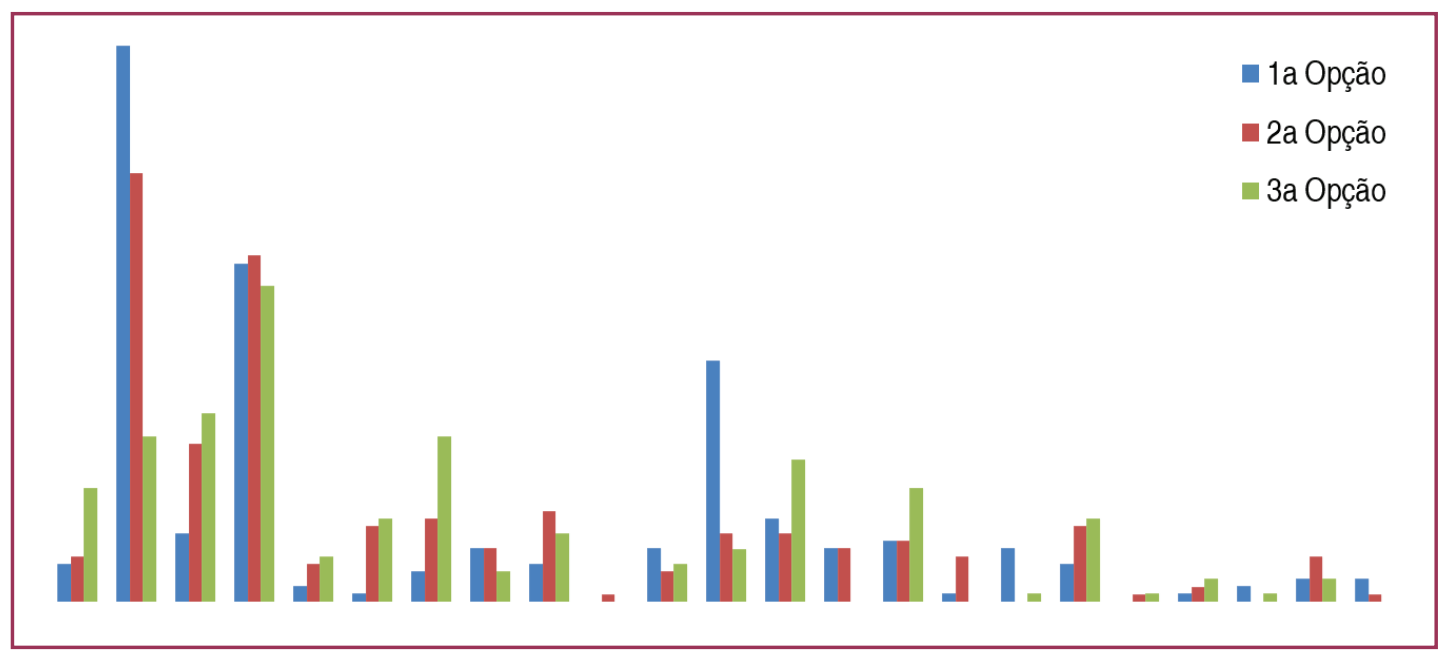

Fonte: Elaborado pelos autores

Em face do grande número de atividades relacionadas, as consolidamos em três categorias, que nomeamos como "Atividades físicas", "Atividades solitárias" e "Atividades sociais". A primeira compreendeu a prática de esportes, ir à praia, dançar e correr. A segunda, utilização da internet, jogos eletrônicos, cinema, ler, filmes, descansar, televisão, música e fotografia. A última compreendeu sair com amigos, ficar com a família, ir a uma discoteca, namorar, divertir-se e viajar, cozinhar, dançar, ir a bar, festas e igreja. Resultados desta nova forma de ver os dados mostram que as atividades sociais aparecem na primeira posição (41\%), seguidas das atividades esportivas (38,6\%) e solitárias (20,1\%), e esta última categoria aparece em primeiro lugar somente quando é citada como terceira opção (Figura 2). 
Figura 2 - Porcentagem das atividades realizadas no tempo livre por categorias

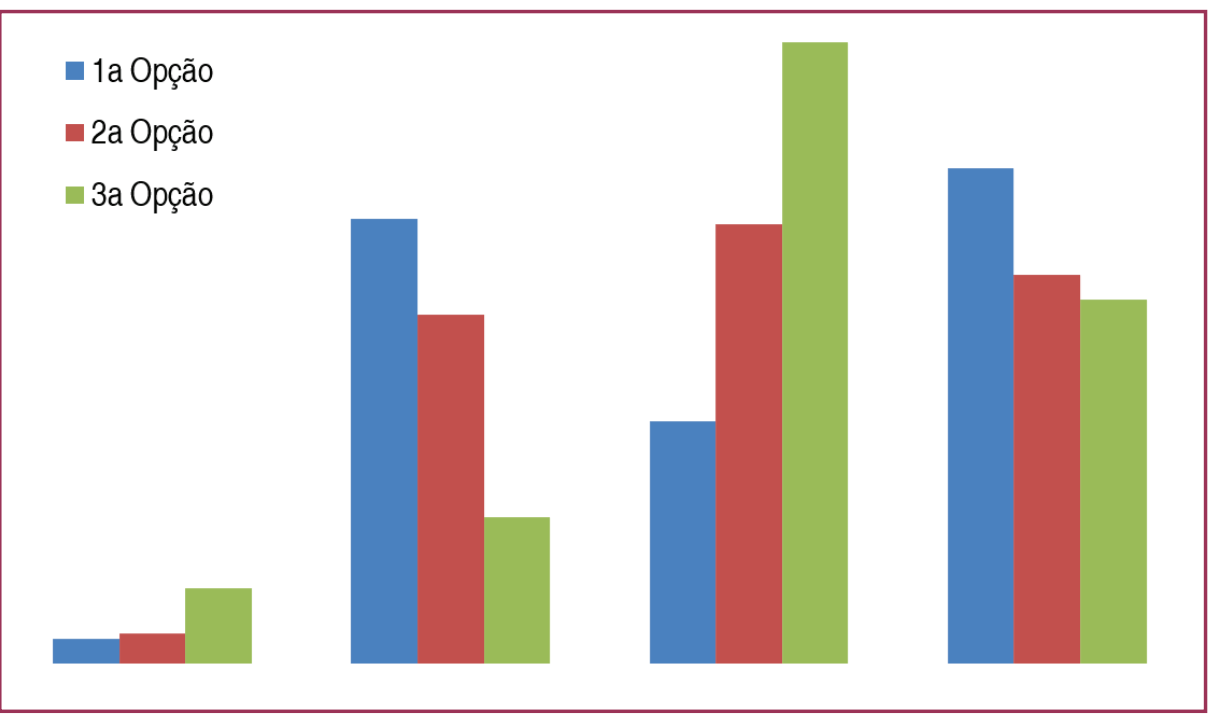

Fonte: elaborado pelos autores

\subsection{0 uso de álcool e outras drogas}

Foi avaliado o uso do álcool e tabaco, as duas drogas que têm permissão legal para serem vendidas, e as seguintes drogas com venda proibida em ambos os países: cocaína, heroína, maconha, crack e haxixe. A Tabela 1 mostra a frequência de uso dos estudantes por país de origem.

Tabela 1 - Frequência absoluta e relativa da resposta positiva ao uso de drogas legais e ilegais dos estudantes brasileiros e portugueses.

\begin{tabular}{|c|c|c|c|c|c|c|}
\hline & \multicolumn{2}{|c|}{ Brasil } & \multicolumn{2}{|c|}{ Portugal } & \multicolumn{2}{|c|}{ Total } \\
\hline & $f$ & $\%$ & $f$ & $\%$ & $f$ & $\%$ \\
\hline Álcool & 55 & 41,0 & 79 & 59,0 & 134 & 56,8 \\
\hline Tabaco* & 31 & 30,4 & 71 & 69,6 & 102 & 42,7 \\
\hline Cocaína & 6 & 66,7 & 3 & 33,3 & 9 & 3,8 \\
\hline Heroína & 1 & 50,0 & 1 & 50,0 & 2 & 0,8 \\
\hline Maconha & 10 & 26,3 & 28 & 73,7 & 38 & 15,9 \\
\hline Crack & 0 & 0,0 & 1 & 100,0 & 1 & 0,4 \\
\hline Haxixe & 5 & 25,0 & 15 & 75,0 & 20 & 8,4 \\
\hline
\end{tabular}

As duas drogas mais consumidas são as que têm venda legalizada, 0 álcool e 0 tabaco. Em relação à primeira droga o seu uso foi declarado por $56,8 \%$ dos estudantes, que responderam "Sim" à questão "Você bebe?". Não há diferença estatística entre os porcentuais de consumo entre os participantes dos dois países. O consumo de álcool também foi avaliado através do instrumento AUDIT, no qual somente $21,3 \%$ dos estudantes relataram não ter feito uso de álcool no último ano. Esta diferença pode ser entendida pelo fato de que no AUDIT $49,4 \%$ deles pontuaram entre 1 e 7 , indicando um beber moderado, o que para os jovens é praticamente considerado como não beber. 
Ainda em relação ao resultado do AUDIT, $29,3 \%$ dos estudantes pontuaram 8 ou mais neste teste, indicando, no mínimo, um padrão de beber excessivo. Comparativamente, 41,5\% dos alunos brasileiros estão neste padrão, enquanto entre os portugueses este percentual é de $21,4 \%$, evidenciando uma diferença significante $\left(\chi^{2}=11,136, p=0,001\right)$. Análise de variância, tendo país e sexo como variáveis independentes e a pontuação do AUDIT como dependente, mostra que os rapazes brasileiros apresentam uma pontuação média maior do que os portugueses $\left(F_{3,237}=18,399, p=0,001\right)$ e que os rapazes bebem mais do que as moças, independente do país de origem $\left(F_{3,237}=13,101, p=0,001\right)$ (Figura 3$)$, mas não há interação significante entre país e sexo.

Figura 3 - Média de pontuação no AUDIT por país e sexo.

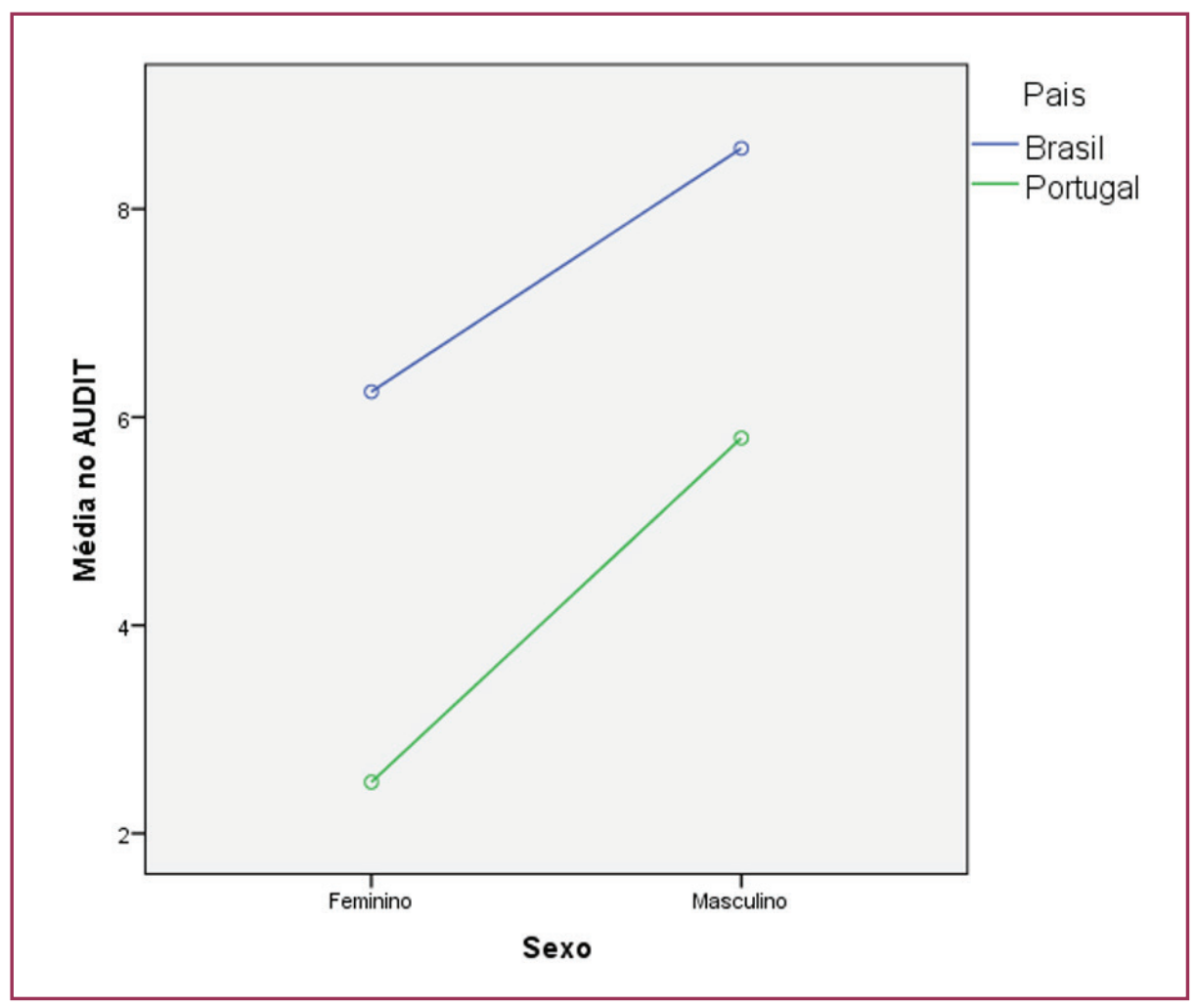

Fonte: Elaborado pelos autores

Beber se embriagando (binge drinking), medido pela questão 3 do AUDIT ("Com que frequência você toma seis ou mais doses em uma ocasião?"), é um comportamento mais presente em jovens, que geralmente bebem nos finais de semana, mas com este padrão de beber excessivo, que é associado a consequências negativas, como baixo rendimento escolar e acidentes (CARDOSO et al., 2015; MARTINIC; MEASHAM, 2008). Como um todo, 43,5\% dos alunos apresentam este comportamento e como no resultado geral do AUDIT os estudantes brasileiros apresentam de forma significante uma prática maior deste comportamento em relação aos portugueses $\left(\chi^{2}=19,074, p=0,001\right)$, especialmente nos níveis mais elevados ("Semanalmente" e "Todos ou quase todos os dias") (Figura 4).

Com relação ao consumo de bebidas alcoólicas, 28,7\% dos brasileiros declararam beber cerveja, ao passo que $15,9 \%$ dos portugueses também o fazem. Não obstante os brasileiros tenham declarado consumir mais cerveja, os jovens portugueses afirmaram fazer maior consumo de vinho, sendo um consumo de $10,3 \%$ dos portugueses e $4,3 \%$ dos brasileiros. 0 
consumo de bebidas destiladas não representou diferença significativa entre ambos os grupos, sendo bastante menor que o consumo de cerveja.

Figura 4 - Porcentagem de alunos que bebem se embriagando por país.

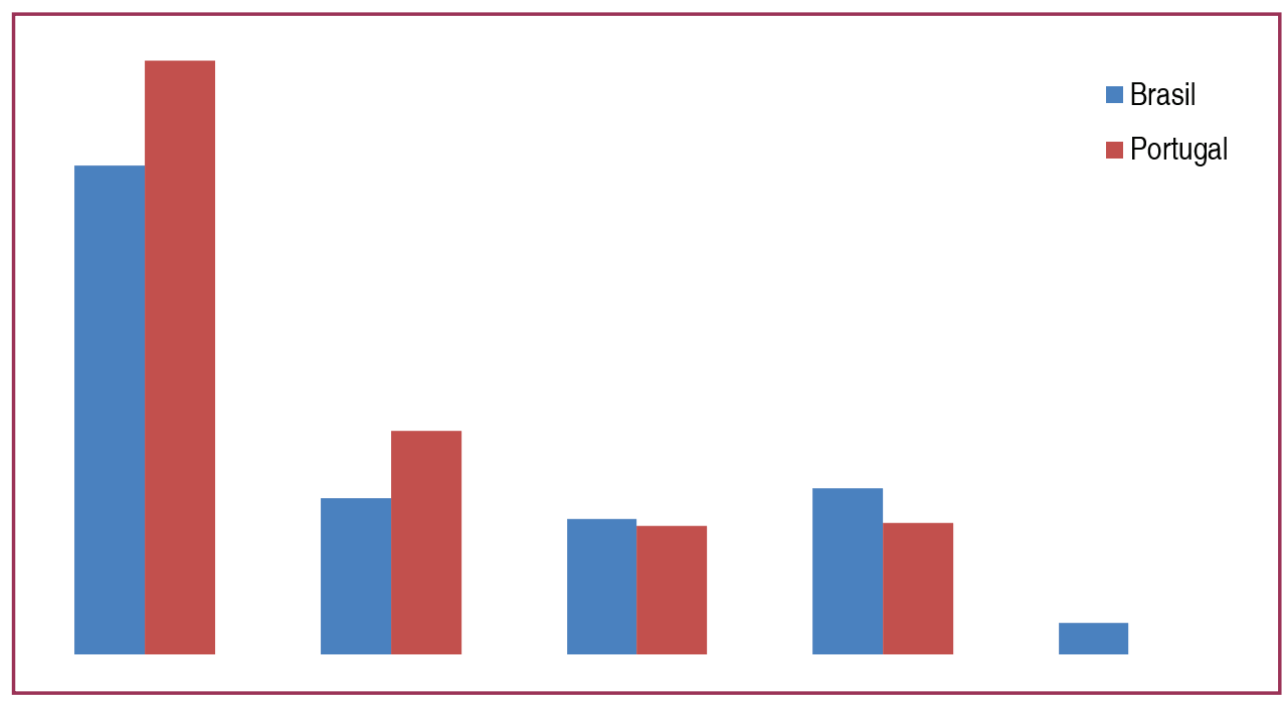

Fonte: elaborado pelos autores

Estudo desenvolvido por Lomba et al. (2011) analisou o comportamento de 1257 jovens em nove cidades portuguesas e apontou que 59,44\% dos jovens entrevistados elegem como fator decisório na escolha pelos locais de diversão noturna a acessibilidade a bebidas alcoólicas com preços mais baixos. Measham e Brain (2005) sugerem que houve um aumento no consumo de bebidas alcoólicas a partir da ampliação do espectro social das juventudes. Para os autores, as escolhas sobre o que e onde beber se tornaram mais amplas com 0 crescimento da indústria de bebidas alcóolicas e das suas estratégias de marketing.

O estudo de Lomba et al. (2011) aponta o álcool como a substância psicoativa mais consumida pelos jovens, seguida da cannabis (maconha) e cocaína. De acordo com o referido estudo, o álcool é consumido por $80 \%$ dos jovens entrevistados seguidos de $40,28 \%$ de consumidores de cannabis nas cidades de Lisboa e Coimbra, dois grandes centros universitários de Portugal.

Estudo com universitários brasileiros e portugueses mostram resultados similares para ambos os países. Ao investigar alunos da Universidade de Aveiro (Portugal), Rodrigues, Salvador, Lourenço e Santos (2014) encontraram que 42,0\% deles relataram ter praticado binge drinking, salientando que nesse estudo os pesquisadores definiram este comportamento como beber até cinco doses por episódio para homens e quatro para mulheres. Resultados semelhantes são encontrados entre universitários brasileiros (FERRAZ; REBELATTO; SCHNEIDER; ANZOLIN, 2017; PARENTE et al., 2017). Entretanto, estudos em outros países se atentam ao fato de os jovens não perceberem seu padrão de uso de álcool como excessivo, extremo ou binge, o que para alguns autores pode significar uma nova cultura de intoxicação, muito mais centrada da gratificação pessoal, na diversão e no sentimento de pertencimento e socialização, trazendo uma forma de uso com caráter hedônico, como possível consequência dos anseios provocados pela sociedade atual de consumo (ANDER; ABRAHAMSSON; BERGNEHR, 2017; EGGINTON; WILLIAMS; PARKER, 2002; MEASHAM; BRAIN, 2005; MEASHAM; ØSTERGAARD, 2011) 
O tabaco, a segunda droga mais usada pelos participantes deste estudo, foi reportado por $42,7 \%$ deles, sendo que entre os portugueses alcançou $49,0 \%$ dos estudantes, contra $33,0 \%$ de brasileiros, diferença esta significante $(\chi 2=5,958, p=0,015)$. Quanto às drogas ilegais, a maconha é citada por $15,9 \%$ dos participantes, seguida do haxixe $(8,4 \%)$. As outras três drogas investigadas tiveram baixos índices de adesão, especialmente a heroína e o crack, com menos de 1,0\% de estudantes usuários delas (Tabela 1).

Com a finalidade de compreender melhor o consumo de drogas ilegais, os estudantes que declaram o uso de pelo menos uma delas foram nomeados como positivos em relação ao uso dessas drogas. Resultados mostram que estes usuários apresentam uma pontuação maior no AUDIT em relação aos não usuários, em que os primeiros apresentam média de 9,24 (DP = $6,37)$ contra 4,59 (DP = 5,81) dos não usuários $\left(F_{1,237}=21,025, p=0,001\right)$.

Em relação ao consumo elevado de bebidas alcoólicas entre universitários, podemos entender que não é um comportamento novo, isto é, algo que tenha começado quando ingressaram na universidade. No Brasil, estudo de Martins et al. (2008) mostra que essa aprendizagem ocorre no ensino médio e a mesma situação é apontada por Paupério, CorteReal, Dias e Fonseca (2012), com alunos de nível equivalente ao ensino médio brasileiro, em Portugal, que encontraram alto consumo de bebidas alcoólicas entre os alunos de cursos que antecedem 0 ingresso na universidade.

Os jovens brasileiros apontaram maior adesão a uma religião do que os portugueses, com diferença significante, $91,3 \%$ dos universitários brasileiros declararam ter religião, ao passo que portugueses afirmaram $58,6 \%$ de adesão a alguma manifestação religiosa, sendo que em ambos os grupos há uma prevalência da religião católica $\left(\chi^{2}=29,388, p=0,001\right)$. Estudos de Sanchez, Opaleye, Chaves, Noto e Nappo (2011) e Silva et al. (2006) discutem os fatores protetivos para o não uso de drogas e destacam o vínculo religioso como um dos possíveis fatores de proteção. De acordo com estudo de Sanchez, Oliveira e Nappo (2008), a religiosidade aparece como segundo principal fator de proteção, tanto para usuários quanto para não usuários, e afirma que a religião se apresenta como sendo importante para a não iniciação ao uso e também para a redução do consumo.

Neste estudo a religião não se mostrou fator protetivo para os estudantes brasileiros; um fator que pode estar interferindo no consumo é a publicidade de bebidas alcoólicas. Os resultados mostram que os brasileiros se declararam mais expostos à publicidade televisiva. Resultado significante, com $83 \%$ deles afirmando sofrer o impacto de algum tipo de publicidade de bebidas, enquanto $69 \%$ dos portugueses afirmaram perceber-se expostos a tais publicidades $(\chi 2=5,891, p=0,015)$. Enfatizando que a principal forma de publicidade é a televisiva, sobre a qual os jovens brasileiros sofrem maior impacto, os estudos de Pinsky e El Jundi (2008) ressaltam a intensidade de uso de situações de lazer veiculadas em publicidade de cerveja, especialmente direcionada ao público jovem.

Estudo de Romera (2014) realizado a partir de análise de folders de publicidade de festas dirigidas a jovens universitários e propagadas nos murais das universidades verificou a intensa proximidade entre vivência de lazer e consumo de cerveja entre os jovens brasileiros e detectou estímulos ao abuso do consumo em cartazes de publicidade em algumas universidades brasileiras. Ao analisar a relação entre publicidade de cerveja e o consumo, Vendrame e Pinsky (2011) observaram que o conteúdo das mensagens publicitárias atua nos processos de decisão do indivíduo, concretizando o consumo. 
É possível que tal relação ocorra devido à maior regulação de publicidade referente ao álcool, algo que não ocorre com as cervejas. Importante destacar que, na mídia brasileira, a publicidade de vinho ou de bebidas destiladas passa por maior controle e rigorosidade frente às imagens veiculadas e horários de veiculação. No entanto, a publicidade de cerveja, que não é atingida pela referido controle e rigorosidade, aparece, muitas vezes, de forma agressiva e bastante convincente, atrelando os contextos e experiências de lazer ao consumo de forma quase que condicionada (OLIVEIRA; ROMERA; MARCELLINO, 2011; PINSKY; EL JUNDI, 2008). $O$ consumo de destilados ou bebidas brancas não apresentou diferença significativa, sendo de $28,7 \%$ para brasileiros e de $29,7 \%$ para os portugueses.

Outra questão relacionada ao uso de bebidas alcoólicas refere-se à condução de veículos automotores, que é uma das grandes preocupações dos gestores de trânsito e de saúde pública. Gorgulho e Ros (2006) argumentam que as estatísticas que relacionam esse uso a acidentes automobilísticos no Brasil são altas, cerca de 70\%, apesar de existirem iniciativas voltadas para a prevenção desse fenômeno. Neste estudo somente $11 \%$ dos estudantes que pontuaram entre zero e 7 no AUDIT dirigiram após beber, porcentagem que aumenta para $36,6 \%$ dos que pontuaram 8 ou mais. Análise estatística mostra que essa diferença é significante $(\chi 2=20,488, p=0,001)$.

Estudo de Romera, Martins e Reis (2017) com jovens torcedores de futebol apontou índices de $36,6 \%$ de sujeitos pontuando acima de 8 no AUDIT, sinalizando o comprometimento com o beber. Esses jovens também se declararam expostos à publicidade de cerveja, que no Brasil está bastante vinculada ao futebol e aos inúmeros campeonatos.

Nesta pesquisa foi também perguntado aos jovens se tinham participado de algum programa preventivo em relação ao uso de drogas quando estavam na Educação Básica. Os resultados apontaram que $57,6 \%$ dos estudantes brasileiros participaram de algum tipo de programa de prevenção escolar, enquanto $39,4 \%$ dos universitários portugueses também o fizeram. Analisando o tipo de programa de que participaram, encontramos que entre os brasileiros predominaram as palestras e, entre os portugueses, predominaram orientações desenvolvidas por professores diretamente aos alunos. 0 tipo de intervenção pode estar relacionado ao menor número de estudantes portugueses pontuando 8 ou mais no AUDIT, indicando um consumo mais moderado de bebidas.

\subsection{As relações entre tempo livre e o uso de álcool e outras drogas}

As comparações entre tempo livre e uso de álcool e outras drogas foram realizadas utilizando-se as três categorias de atividades que os estudantes praticam, que foram nomeadas como "Atividades físicas", "Atividades solitárias" e "Atividades sociais" (Figura 2), posteriormente cruzadas com o resultado do AUDIT, padrão de beber se embriagando (binge drinking) e utilização de drogas ilegais. Os resultados não mostraram nenhuma associação estatística entre essas variáveis, e que, para este público jovem, usar bebidas alcoólicas, tabaco ou drogas ilegais aparentemente não interfere nas atividades que praticam durante 0 tempo livre de que dispõem.

Entretanto, pesquisa realizada no Brasil por Gorgulho e Ros (2006), posteriormente compilada por Martinic e Measham (2008), que compreendia grupos focais com jovens de diversos países, apontou que algumas atividades sociais, como "sair à noite" e "sair com os 
amigos", estavam intrinsecamente relacionadas com o consumo de bebidas alcóolicas para os jovens brasileiros. Em concordância, Measham e Østergaard (2011) argumentam que 0 processo de intoxicação não é uma simples escolha individual, mas, sim, um comportamento que é apreendido a nível social e cultural. Possivelmente os índices de atividades sociais e esportivas tenham relação direta com os altos níveis de consumo de bebidas, muito embora a prática esportiva não tenha representado fator de proteção para o não consumo, especialmente de bebidas por parte dos estudantes de ambos os países.

\section{CONCLUSÕES}

Resultados mostram que o grupo estudado é muito ativo, pois quase $80 \%$ deles praticam algum tipo de esporte, e que utilizam o seu tempo livre em 22 atividades diferentes, que foram categorizadas em esportivas, sociais e solitárias. Na primeira opção de atividade aparecem as sociais, seguidas das esportivas e, por último, as solitárias.

Esses estudantes fazem uso principalmente das drogas legais, álcool e tabaco, com os brasileiros fazendo maior uso de bebidas alcoólicas e os portugueses de tabaco. As drogas ilegais mais consumidas são a maconha e o haxixe. O beber e dirigir é citado mais pelos jovens que apresentam padrão de beber excessivo, que é indicado pela pontuação 8 ou mais no teste AUDIT.

Finalizando, os resultados mostram estudantes com um padrão de beber excessivo, especialmente entre os brasileiros, e a utilização de bebidas alcoólicas e drogas ilegais como um acompanhamento destas formas de utilização do tempo livre.

\section{REFERÊNCIAS}

ALDRIDGE, Judith; MEASHAM, Fiona; WILLIAMS, Lisa. Illegal leisure revisited: Changing patterns of alcohol and drug use in adolescents and young adults. London: Routledge, 2013.

ANDER, Birgitta; ABRAHAMSSON, Agneta; BERGNEHR, Disa. "It is ok to be drunk, but not too drunk": party socialising, drinking ideals, and learning trajectories in Swedish adolescent discourse on alcohol use. Journal of Youth Studies, v. 20, n. 7, p. 841-854, 2017.

BABOR, Thomas F. et al. AUDIT: The Alcohol Use Disorders Identification Test: Guidelines for Use in Primary Care. $2^{\text {nd }}$. ed. Geneva: World Health Organization, 2001.

BAUMAN, Zygmunt. Amor líquido: sobre a fragilidade dos laços humanos. Rio de Janeiro: Jorge Zahar, 2003.

CALDWELL, Linda; SMITH, Edward A. Leisure as a Context for Youth Development and Delinquency Prevention. Australian and New Zealand Journal of Criminology, v. 39, n. 3, p. 398-418, 2006.

CARDOSO, Fernanda Mourão et al. Fatores associados à prática do binge drinking entre estudantes da área da saúde. Revista CEFAC, v. 17, n. 2, p. 475-484, abr. 2015.

EGGINTON, Roy; WILLIAMS, Lisa; PARKER, Howard. Going out drinking: The centrality of heavy alcohol use in English adolescents' leisure time and poly-substance-taking repertoires. Journal of 
FERRAZ, Lucimare et al. The use of alcohol and tobacco among students of a university in Southern Brazil. Revista Brasileira em Promoção da Saúde, v. 30, n. 1, p.79-85, 2017.

GORGULHO, Mônica; DA ROS, Vera. Alcohol and harm reduction in Brazil. International Journal of Drug Policy, v. 17, n. 4, p. 350-357, 2006.

LIPOVETSKY, Gilles. A felicidade paradoxal: ensaios sobre a sociedade de hiperconsumo. Trad. Maria Lúcia Machado. São Paulo: Companhia das Letras, 2007.

LOMBA, Lurdes et al. Jovens portugueses que frequentam ambientes recreativos nocturnos: Quem são e comportamentos que adoptam. Revista Toxicodependências v.17, n. 1, p. 3-15, 2011.

MARTINIC, Marjana; MEASHAM, Fiona (Org.). Swimming with crocodiles: the culture of extreme drinking. New York: Routledge, 2008.

MARTINS, Raul Aragão et al. Utilização do Alcohol Use Disorders Identification Test (AUDIT) para identificação do consumo de álcool entre estudantes do ensino médio. Interamerican Journal of Psychology, v 42, n 2, p. 307-316, 2008.

MEASHAM, Fiona; BRAIN, Kevin. "Binge" drinking, British alcohol policy and the new culture of intoxication. Crime, Media, Culture, v. 1, n. 3, p. 262-283, 2005.

MEASHAM, Fiona; ØSTERGAARD, Jeanette. The Phenomenon of Youth Drinking. In: SAUNDERNS, J.P; REY, J.M. (Org.). Young People and Alcohol. Chichester: John Wiley. 2011. p. 1-17.

MÉNDEZ, Eduardo. Uma versão brasileira do AUDIT (Alcohol Use Disorders Identification Test). $121 f$. 1999. Dissertação (Mestrado) - Universidade Federal de Pelotas, Pelotas, 1999.

MIELKE, Grégore Iven et al. Atividade física e fatores associados em universitários do primeiro ano da Universidade Federal de Pelotas. Revista Brasileira de Atividade Física \& Saúde, v. 15, n. 1, p. 57-64, 2010.

OLIVEIRA, Marcela; ROMERA, Liana Abrão; MARCELLINO, Nelson Carvalho. Lazer e juventude: análise das propagandas de cerveja veiculadas pela televisão. Journal of Physical Education, v. 22, n. 4, p. 535-546, 2011.

PARENTE, Ezequiel Aguiar et al. Alcohol use among medical students: a possible risk for future doctors? Uso de Álcool entre Estudantes de Medicina: um possível risco para futuros médicos?. Journal of Health \& Biological Sciences, v. 5, n. 4, p. 311-319, 2017.

PAUPÉRIO, Tiago; CORTE-REAL, Nuno; DIAS, Claudia, FONSECA, António, Sport, substance use and satisfaction with life: What relationship? European Journal of Sport Science, v.12, n.1, p. 73-80, 2012.

PEREIRA, Júlio César Rodrigues. Análise de dados qualitativos: estratégias metodológicas para as ciências da saúde humanas e sociais. São Paulo: Edusp, 1999.

PINSKY, llana; EL JUNDI, Sami. O impacto da publicidade de bebidas alcoólicas sobre o consumo entre jovens: Revisão da literatura internacional. Revista Brasileira de Psiquiatria, v. 30, n. 4, p. 362374, 2008.

RODRIGUES, Pedro et al. Padrões de consumo de álcool em estudantes da Universidade de Aveiro: Relação com comportamentos de risco e stress. Análise Psicológica, v. 32, n. 4, p. 453-466, 2014. 
ROMERA, Liana Abrão. Lazer e festas: Estudo sobre os modos de divulgação de bebidas nos campi universitários. Cadernos Brasileiros de Terapia Ocupacional da UFSCar, v. 22, n. Supl. Esp., p.95102, 2014.

ROMERA, Liana Abrão; MARTINS, Raul Aragão; REIS, Heloísa Helena Baldy dos. Torcedores Jovens e Padrão de Consumo de Bebidas Alcoólicas: uma Modalidade de Lazer. LICERE-Revista do Programa de Pós-graduação Interdisciplinar em Estudos do Lazer, v. 20, n. 1, p. 181-200, 2017.

SANCHEZ, Zila Van der Meer et al. God forbids or mom disapproves? Religious beliefs that prevent drug use among youth. Journal of Adolescent Research, v. 26, n. 5, p. 591-616, 2011.

SANCHEZ, Zila Van der Meer; OLIVEIRA, Lucio Garcia de; NAPPO, Solange Aparecida. Religiosity as a protective factor against the use of drugs. Substance use \& misuse, v. 43, n. 10, p. 1476-1486, 2008.

SILVA, Leonardo et al. Fatores associados ao consumo de álcool e drogas entre estudantes universitários. Revista de Saúde Pública, v. 40, p. 280-288, 2006.

SPSS INC. SPSS base 14.0 user's guide. Prentice Hall, 2005.

VENDRAME, Alan; PINSKY, Ilana. Ineficácia da autorregulamentação das propagandas de bebidas alcoólicas: uma revisão sistemática da literatura internacional. Revista Brasileira de Psiquiatria, v. 33, n. 2, p. 196-202, jun. 2011. 